
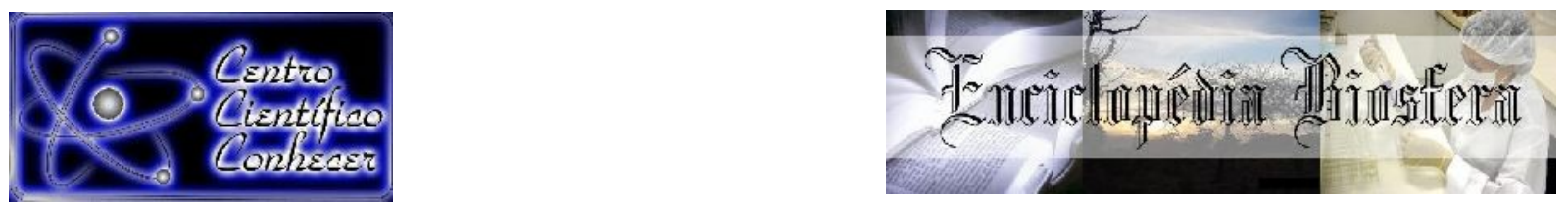

\title{
MACROFUNGOS ENCONTRADOS NO CAMPUS X DA UNIVERSIDADE DO ESTADO DA BAHIA
}

\author{
Lorrane Rocha da Silva ${ }^{1}$, Jorge Luiz Fortuna ${ }^{2}$ \\ ${ }^{1}$ Licenciadas em Ciências Biológicas pela Universidade do Estado da Bahia (UNEB), \\ Campus X, Teixeira de Freitas-BA. \\ ${ }^{2}$ Professor Adjunto da área de Microbiologia do curso de Ciências Biológicas pela \\ Universidade do Estado da Bahia (UNEB), Campus X, Teixeira de Freitas-BA. \\ E-mail: jfortuna@uneb.br
}

Recebido em: 15/11/2020 - Aprovado em: 15/12/2020 - Publicado em: 30/12/2020
DOI: 10.18677/EnciBio_2020D24

\begin{abstract}
Os fungos constituem o mais numeroso grupo de seres vivos existentes excluindo-se os insetos. Os macrofungos são fungos que formam estruturas reprodutivas macroscópicas, ou seja, que podem ser vistas a olho nu, sendo popularmente conhecidos como cogumelos e orelhas-de-pau, podendo ser parasitas, sapróbios e micorrízicos. Com o acréscimo do desmatamento na região do Extremo Sul da Bahia, faz-se necessário, a elaboração de trabalhos sobre identificação e diversidade dos macrofungos existentes no Extremo Sul da Bahia, para além das áreas de reservas ambientais. Este trabalho teve como objetivo coletar e identificar macrofungos encontrados na área do Campus $X$ da Universidade do Estado da Bahia (UNEB), no município de Teixeira de Freitas-BA, localizado no Extremo Sul da Bahia. As coletas foram realizadas continuamente entre os meses de maio e julho de 2017. Os fungos foram coletados nas proximidades dos pavilhões 1, 2, 3 e próximo ao estacionamento da universidade. Foram identificados 14 macrofungos, sendo 13 (92,86\%) do filo Basidiomycota e apenas um (7,14\%) do filo Ascomycota. Os fungos basidiomicotas foram agrupados nas seguintes famílias: Agaricaceae, Sclerodermataceae, Polyporaceae, Hymenochaetaceae, Psathyrellaceae, Inocybaceae e Entolomataceae. O filo Ascomycota foi identificado apenas um espécime da família Geoglossaceae. Com os resultados obtidos pode-se ressaltar a importância de trabalhos de identificação de macrofungos, para que assim os registros de espécies de macrofungos no Extremo Sul aumentem podendo, assim, ter um mapeamento de macrofungos endêmicos, bem como macrofungos anuais da região.
\end{abstract}

RESUMO

PALAVRAS-CHAVE: Ascomycota, Basidiomycota, Fungos. 


\title{
IDENTIFICATION OF MACROFUNGI FOUND ON CAMPUS X OF THE STATE UNIVERSITY OF BAHIA
}

\begin{abstract}
Fungi constitute the most numerous groups of living beings in existence, excluding insects. Macrofungi are fungi that form macroscopic reproductive structures, therefore allowing them to be visualized, being popularly known as mushrooms and wood-earmushrooms, and can be parasites, saprobics and mycorrhizals. With the increased deforestation of the region of the Southernmost of Bahia, it is necessary to develop a work on the identification and diversity of macrofungi existing in this region, in addition to the areas of environmental reserves. The objectives of this study was to collect and identify macrofungi found in the area of the Campus $X$ of the State University of Bahia (UNEB), in the municipality of Teixeira de Freitas, BA, Brazil, which is located in the Southernmost of Bahia. The samples were collected continuously between May and July 2017, and were collected in the vicinity of pavilions 1,2,3, and near the university parking lot. A total of 14 macrofungi were identified, of which $13(92.86 \%)$ belonged to phylum basidiomycota and only one $(7.14 \%)$ to the phylum Ascomycota. The fungi basidiomycota were grouped into the following families: Agaricaceae, Sclerodermataceae, Polyporaceae, Hymenochaetaceae, Psathyrellaceae, Inocybaceae and Entolomataceae. The phylum Ascomycota presented only one specimen of the family Geoglossaceae. The obtained results allow to highlight the importance of macrofungal identification studies, in a way to increase the records of macrofungal species in the Southernmost of Bahia, thus allowing the mapping of endemic and annual macrofungi occurring in the region.
\end{abstract}

KEYWORDS: Ascomycota, Basidiomycota, Fungi.

\section{INTRODUÇÃO}

Os fungos são seres eucariontes aclorofilados pertencentes ao reino Fungi (GIMENES; MATHEUS, 2010). Este reino é exclusivo para esses organismos, no entanto, anteriormente os fungos pertenciam ao reino Plantae. Com o avanço da tecnologia, como as técnicas de microscopia e bioquímica, os fungos foram aproximados aos animais (TERÇARIOLI et al., 2010), assim sendo, transferidos para um reino exclusivamente dos fungos.

Segundo Silva (2013) excluindo-se os insetos, os fungos constituem o mais numeroso grupo de seres vivos existentes. Apesar de estarem em quase todos os ambientes, a diversidade dos fungos ainda constitui um grande desafio para os cientistas, mesmo em locais aparentemente bem estudados (MARQUES, 2012). O Brasil possui grande parte da diversidade fúngica (PIRES et al., 2014). Pires et al. (2014) afirmam que os fungos são relativamente pouco estudados no Brasil, os estudos se concentram em pequenas extensões do território brasileiro, não trazendo um ponto positivo para o conhecimento da diversidade fúngica brasileira. Estima-se que exista 1,5 milhão de espécies de fungos, porém, há apenas 90 mil espécies descritas (ESPOSITO; AZEVEDO, 2010).

Os fungos são separados em microfungos e macrofungos. Os microfungos são popularmente conhecidos como bolores e mofos, sendo encontrados em diversos substratos, tais como pães, frutos, couro, paredes, etc. (ESPOSITO; AZEVEDO 2010). 
Apesar de sua importância, as pessoas relacionam os fungos apenas a problemas de saúde, deterioração de alimentos e objetos. Quando na verdade estão presentes no seu cotidiano para seu benefício, como as leveduras que são fungos unicelulares, que fazem fermentação, usadas na fabricação de pães, massas e na produção de bebidas como cerveja e vinho (SILVA; COELHO, 2006; GIMENES; MATHEUS, 2010). Outros fungos podem ser úteis na indústria farmacêutica como alguns do gênero Penicillium e Cephalosporium. Seus compostos podem ter ação antibacteriana, antifúngica, antiviral e antiblástica (GONÇALVES, 2010).

Os macrofungos são fungos que formam estruturas reprodutivas macroscópicas, ou seja, que podem ser vistas a olho nu, sendo popularmente conhecidos como cogumelos e orelhas-de-pau, podendo ser parasitas, sapróbios e micorrízicos (GIMENES; MATHEUS, 2010). Estes podem ser comestíveis, alucinógenos, bem como ser usados como antimicrobianos. Segundo Domann et al. (2015), os macrofungos contém naturalmente componentes antimicrobianos que possibilitam a sua sobrevivência no ambiente. Assim com a possibilidade de combater bactérias que podem vir a causar doenças.

Os fungos também são responsáveis pela produção de importantes ácidos orgânicos, produção de enzimas de valor industrial e de elevado valor econômico, destacando as celulases, lacases, etc., também pelo controle biológico de insetospragas (ESPOSITO; AZEVEDO 2010). Também são importantes para os vegetais, principalmente na ação micorrízicas. As micorrizas são muito utilizadas na agricultura como um recurso mais barato que favorece a absorção de um nutriente ou mais, que a planta não conseguiria absorver em quantidade, isso traria prejuízos a mesma, como desenvolvimento de caules e folhas, as micorrizas agem em ação mutualística com as plantas, ambas se beneficiando (COSTA et al., 1989).

Os fungos degradadores, disponibilizam as substâncias adquiridas nessa degradação para outros organismos. Esses são responsáveis por um dos mais importantes ciclos do carbono na natureza (ESPOSITO; AZEVEDO 2010). Por tanto as atividades dos fungos na natureza são tão necessárias para a continuidade da existência do planeta, quanto são aquelas desempenhadas pelos organismos produtores (SILVA; COELHO, 2006).

Esposito e Azevedo (2010) descrevem sobre a possível extinção em massa dos fungos, baseado em um estudo de 20 anos, onde se notou um acentuado declínio de espécies observadas durante este período, sendo este declínio pode ser explicado pelo acréscimo de poluição e desmatamento.

Dentro deste contexto faz-se necessário a elaboração de trabalhos sobre identificação e diversidade dos macrofungos no Extremo Sul da Bahia. Pois esta região, devido à exploração da madeira da Mata Atlântica, vem sofrendo grandes modificações estruturais e socioeconômicas desde a década de 1970 (ALMEIDA et al., 2008). Sendo assim, registros sobre os macrofungos contidos nessa região tornará possível um levantamento de possíveis espécies endêmicas bem como as espécies que correm risco de extinção, também havendo a possibilidade de reconhecimento de espécies que se adaptaram ao meio urbano.

Portanto, este trabalho teve como objetivo coletar e identificar macrofungos encontrados na área do Campus $X$ da Universidade do Estado da Bahia (UNEB), no município de Teixeira de Freitas-BA, localizado no Extremo Sul da Bahia. 


\section{MATERIAL E MÉTODOS}

A área de estudo se localiza na cidade de Teixeira de Freitas, Extremo Sul da Bahia, no Departamento de Educação da Universidade do Estado da Bahia (UNEB), Campus X. O campus está localizado na área urbana da cidade e possui três pavilhões. A área é relativamente grande, tendo uma área total de $18.594,70 \mathrm{~m}^{2}$, sendo que a área sem ocupação é de $9.682,74 \mathrm{~m}^{2}$ (PROINFRA, 2017), com poucos vegetais de grande porte.

As coletas foram realizadas continuamente entre os meses de maio e julho de 2017, isto é, assim que surgiam os macrofungos eles eram coletados. Os fungos foram coletados nas proximidades dos pavilhões 1, 2, 3 e próximo ao estacionamento da universidade. Os fungos foram coletados principalmente de gramados e troncos de árvores em decomposição, espalhadas nas proximidades dos pavilhões. Cada amostra, antes de serem retirados do seu substrato natural, foi fotografada, bem como realizados registros fotográficos de suas estruturas macroscópicas, que davam para ser observadas naquele momento. Pois pode ocorrer de perderem algumas das características como coloração, tamanho, etc., até chegar ao laboratório e serem fotografados novamente (GIMENES; MATHEUS, 2010).

Foi elaborada uma ficha para identificação de cada fungo contendo as características observadas, como cor do píleo, lamela, estipe e o substrato onde foi encontrado. Em seguida foram retirados do substrato com o auxílio de uma faca, colocados separadamente (ou em conjunto, caso fossem da mesma espécie, encontrada no mesmo substrato) em sacos de papel, para que assim seus esporos não se misturassem com os de outras espécies.

As análises das características macroscópicas foram baseadas nos caracteres diagnósticos propostos na literatura especializada (DENNIS, 1952; PUCCINELI, 2007; CORTEZ; SILVEIRA, 2007a; 2007b; ROTHER; SILVEIRA, 2008; SILVA et al., 2008), feitas a olho nu e para maiores detalhes com 0 auxílio de um microscópio estereoscópico (PEREIRA; PUTZKE, 1990, GUGLIOTA; CAPELARI, 1998). A identificação dos espécimes coletados está de acordo com a nomenclatura do banco de dados do site Mycobank (2017) e Species 2000 (2017). Também foi utilizado o programa MycoKeys 4.0 proveniente de um site internacional, específico para identificação de macrofungos (PETERSEN et al., 2014).

No Laboratório de Biologia dos Fungos, foram realizados registros fotográficos com régua medindo todo o macrofungo (altura, largura, píleo, etc.), para comparação no momento da identificação. Também foi executado um procedimento que possibilita a liberação de esporos dos macrofungos de forma que esses possam ser conservados posteriormente. Esse processo consiste na seguinte forma: os macrofungos maiores ou mais resistentes foram colocados sobre um papel com um furo no meio. O diâmetro do furo no papel depende do diâmetro do estipe e do basidioma. O píleo foi colocado com as lamelas para baixo.

Este papel com o basidioma foi colocado sobre um Becker contendo água, esses foram colocados dentro de um local de temperatura ambiente e escuro, entre 12-24 h, a depender da condição do macrofungo, pois alguns muito frágeis, não suportam ficar por $24 \mathrm{~h}$, pois suas estruturas começam a se desfazer, correndo assim o risco de perda de parte importantes do basidioma (GUGLIOTTA; CAPELARI, 1998). 
Posteriormente, os papeis com as impressões de esporos foram colocados em sacos de papel, separadamente, identificados com a numeração do fungo coletado, e em seguida colocados juntamente com as demais impressões de esporos dentro de um saco plástico. Que foram fechados de forma que os papéis não pudessem ficar úmidos, dessa forma diminuindo a possibilidade de perda dos esporos.

Os demais basidiomas que não foram retirados à impressão de esporos, por não estarem com o chapéu totalmente aberto ou por serem muito pequenos e frágeis, bem como os outros que houve a possibilidade da impressão dos esporos, foram recolocados em envelope de papel e levados a estufa de secagem a uma temperatura de $45^{\circ} \mathrm{C} / 12-24 \mathrm{~h}$, dessa forma tornando-se exsicatas, para que assim possam fazer parte da Micoteca do Laboratório de Biologia dos Fungos.

A partir das impressões de esporos coletas, foram feitas lâminas para a visualização microscópica. As lâminas foram preparadas com água destilada e resina, pois alguns esporos não ficavam visíveis em água ou em resina, por outro lado alguns foram possíveis de serem visualizados nas duas substâncias.

As lâminas foram identificadas com o número do fungo coletado, a substância (água ou resina) e a data em que foi preparada. Alguns fungos, os quais não houve liberação de esporos foi feita uma raspagem entre as lamelas com a alça bacteriológica, em cima da lâmina com a substância (resina ou água). No caso os fungos sem lamelas, foram feitos cortes ou raspagem para tentativa de visualização dos esporos. As lâminas prontas foram levadas o microscópio para visualização, onde os esporos visualizados foram fotografados, nas visualizações do aumento de 400X e 1.000X.

Os fungos coletados, bem como os identificados, foram devidamente secos na estufa para conservação e guardados em pequenas caixas de papel, contendo a identificação e classificação, data e substrato. As impressões dos esporos foram guardadas no freezer para pesquisas posteriores, as lâminas com basidiósporos visualizados foram seladas com esmalte incolor, para que os basidiósporos possam ser conservados por mais tempo, os registros fotográficos realizados foram arquivados. Assim, todo o material ficará disponível para eventuais pesquisadores de macrofungos e consequentemente futuras pesquisas.

\section{RESULTADOS E DISCUSSÔES}

Foram identificados 14 macrofungos, sendo 13 (92,86\%) do filo Basidiomycota e apenas um $(7,14 \%)$ do filo Ascomycota. Os basidiomicotas foram identificados em três ordens: nove (69,23\%) Agaricales (famílias Agaricaceae; Psathyrellaceae; Inocybaceae; Entolomataceae), três (23,08\%) Polyporales (famílias Polyporaceae e Hymenochaetaceae) e apenas um (7,69\%) Boletales (família Sclerodermataceae). O filo Ascomycota foi identificado em apenas uma ordem: Geoglossales (família Geoglossaceae) (Tabela 1).

TABELA 1. Classificação em nível de espécie.

\begin{tabular}{cccc}
\hline ORDEM & FAMÍLIA & ESPÉCIE & $\begin{array}{c}\text { NÚMERO } \\
\text { INDIVÍDUOS }\end{array}$ \\
\hline Agaricales & Agaricaceae & Chlorophyllum molybdites & 07 \\
& Psathyrellaceae & Macrolepiota sp. 1 & 01 \\
& Inocybaceae & Macrolepiota sp. 2 & 02 \\
\hline
\end{tabular}




\begin{tabular}{cccc}
\hline & & Agaricus spp. & 01 \\
& & Panaeolus acuminatus & 05 \\
& & Psathyrella spp. & 04 \\
& & Inocybe rimosa & 05 \\
& & Entoloma serrulatum & 02 \\
& & Entoloma spp. & 01 \\
\hline \multirow{2}{*}{ Polyporales } & Polyporaceae & Lentinus crinitus & 01 \\
& Hymenochaetaceae & Pycnoporus sanguineus & 01 \\
\cline { 2 - 4 } & & Phylloporia chrysita & 05 \\
\hline Boletales & Sclerodermataceae & Scleroderma spp. & 06 \\
\hline Geoglossales & Geoglossaceae & Geoglossum fallax & 10 \\
\hline
\end{tabular}

Os macrofungos também foram identificados em nível de espécie e/ou gênero em suas respectivas famílias e ordens. A ordem Agaricales foi identificada representativamente por 28 indivíduos, sendo que 11 indivíduos pertenciam a família Agaricaeae, que foram identificados em nivel de éspecie (Chlorophyllum molybdite com sete indivíduos), e quatro em nível de gênero (Macrolepiota sp. $1 \mathrm{com}$ um indivíduo; Macrolepiota sp. 2 com dois indivíduos e um Agaricus spp.). A família Psathyrellaceae foi identificada em nove indivíduos com cinco em nível de uma única espécie (Panaeolus acuminatus) e quatro em nível de gênero (Psathyrella spp.).

A família Inocybaceae, foi identificada em cinco indivíduos sendo esses identificados em nível de espécie (Inocybe rimosa). A família Entolomataceae foi identificada em três indivíduos sendo dois em nível de espécie (Entoloma serrulatum) e um em nível de gênero (Entoloma spp.).

A ordem Polyporales foi identificada a partir de sete indivíduos, em duas famílias. $\mathrm{Na}$ família Polyporaceae com dois indivíduos identificados em nível de espécies (Lentinus crinitus e Pycnoporus sanguineus). A família Hymenochaetaceae com cinco indivíduos identificados de uma mesma espécie (Phylloporia chrysita).

A ordem Boletales foi representada com seis indivíduos sendo esses pertencentes a uma única família e identificados em nível de gênero (Scleroderma spp.)

O filo Ascomycota foi representada por dez indivíduos, sendo esses pertencentes a família Geoglossaceae, todos os indivíduos identificados em nível de espécie (Geoglossum fallax).

\section{MACROFUNGOS IDENTIFICADOS}

\section{Família Agaricaceae}

A família Agaricaceae é uma das famílias mais diversas da ordem Agaricales, estima-se que contenha 50 gêneros e 900 espécies. A impressão dos esporos normalmente é branco ou colorido. Também há variações na estrutura do himenóforo e na superfície do píleo (WEBSTER; WEBER, 2007).

Segundo Singer (1986), a família Agaricaceae apresenta o píleo frequentemente umbonado ou convexo, glabro escamoso, furfuráceo, com margem sulcada, estriada ou lisa. Lamelas geralmente livres, às vezes com colário, mais raramente adnexas, adnatas a decorrentes, estipe central, geralmente giboso.

De acordo com Didukh et al. (2003), a família possui espécies que são historicamente e economicamente conhecidas por seus valores nutricionais e 
propriedades farmacológicas.

Chlorophyllum molybdites (BRASIL; Bahia: Teixeira de Freitas; Campus X; Silva, L. R.)

Foram coletados sete indivíduos desta espécie, foram encontrados em uma parte menos frequentada por pessoas, foram encontrados alguns agrupados e outros solitários. Segundo Hernández et al. (2014) é característico desta espécie, além de serem comuns em terras abandonas e ricas em matéria orgânica e por sua característica carnosa pode parecer uma espécie comestível, no entanto é toxica e pode provocar problemas gastrointestinais.

São caracterizados por seu píleo branco com escamas marrons, com anel de curvatura dupla. Os basidiomas encontrados possuem certa de 9,0-10,0 cm de altura e o píleo com 6,0-9,0 cm de diâmetro, têm impressão de esporos na cor verde, o que os tornam facilmente reconhecido (VELLINGA, 2003). Os basidiósporos com cerca de 9,0$11,0 \times 6,0-8,0 \mu \mathrm{m}$, translúcido, com poro germinativo (Figura 1).

FIGURA 1. (1) Chlorophyllum molybdites. (A) Basidiomas; (B) Lamelas verdes; (C) Impressão de esporos; (D) Esporos. (2) Macrolepiota sp. 1. (A) Basidioma; (B) Lamela e anel no estipe. (3) (A) Macrolepiota sp. 2 no substrato; (B) Basidiomas. (4) (A) Agaricus spp. em substrato; (B) Basidioma; (C) Lamelas; (D) Basidiósporos. (5) Scleroderma spp. (A) Fase jovem; (B) Estágio intermediário com basidiósporo abrindo; (C) Basidioma maduro; (D) Basidiósporos. (6) (A) L. crinitus em substrato; (B) Basidioma completo; (C) Píleo umbilicado; (D) Lamelas. (7) (A-B)

Pycnoporus sanguineus no substrato; (C) Himênio poroide; (D) Parte superior do basidioma.

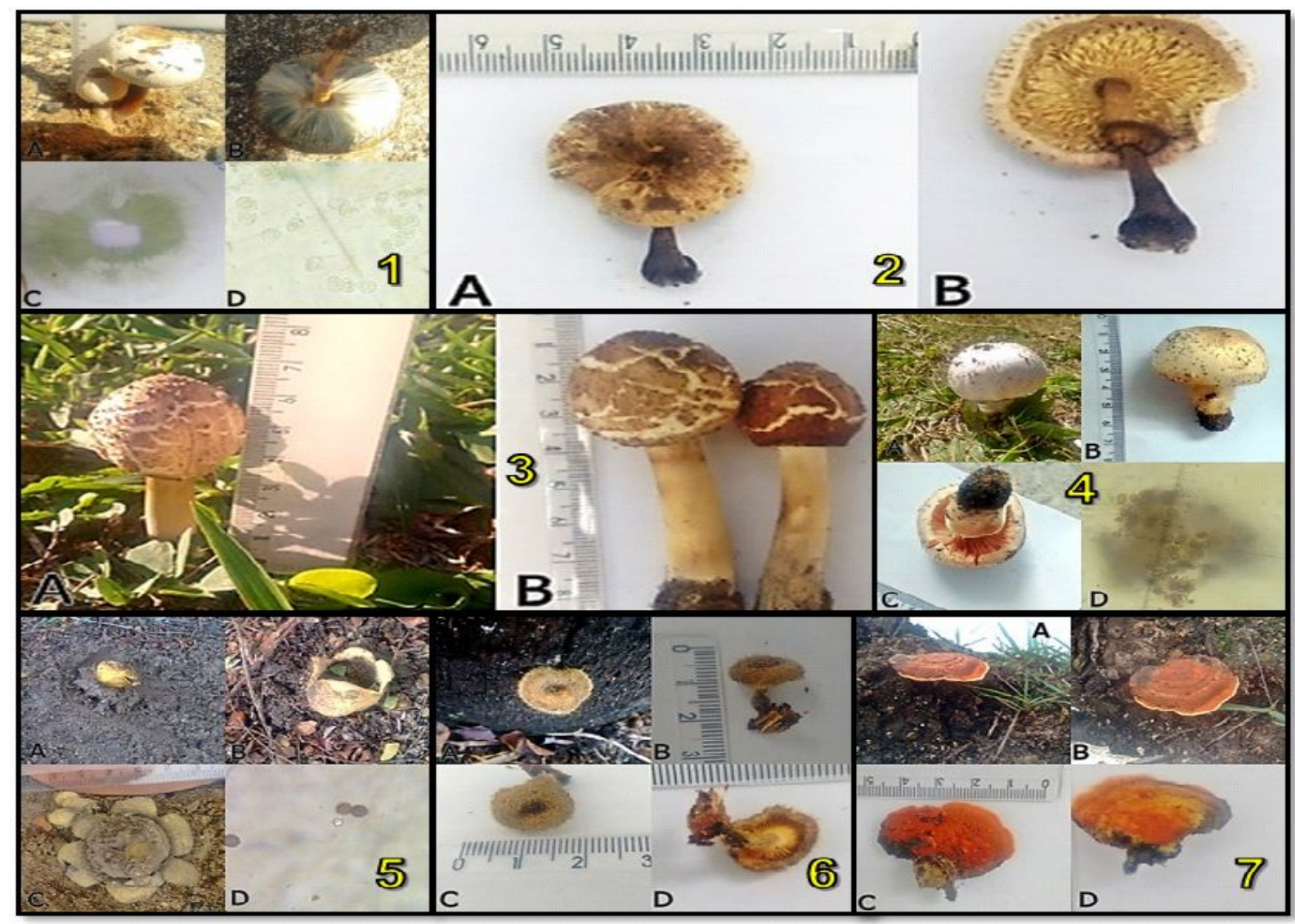

Macrolepiota sp. 1. (BRASIL; Bahia: Teixeira de Freitas; Campus X; Silva, L. R.) 
Foi coletado apenas um individuo da espécie, que já estava em último estágio de vida, lamelas bege, quase brancas, bem separadas, provavelmente por já haver liberado esporos. Medindo cerca de $4,0 \mathrm{~cm}$ altura, estipe centralizado, de cor marrom escuro, com anel. Píleo medindo 3,0-4,0 cm de diâmetro (Figura 1). Webster e Weber (2007) afirmam que crescem em sua maioria, as especies desse genero crescem em parques, pastagens e bosques. Eles são sapróbios. O basidioma de cor castanha pálida geralmente é grande, alguns dessa espécie são considerados coméstiveis, outros podem gerar problemas gastrointestinal. Geralmente as espécies possuem anel bem evidente.

Macrolepiota sp. 2 (BRASIL; Bahia: Teixeira de Freitas; Campus X; Silva, L. R.)

Foram coletados dois espécimes com píleo ainda fechado, provavelmente um espécime imaturo, píleo no formato parabólico, superfície composta por esquamulas marrons, medindo 2,0-3,0 cm de diâmetro. O estipe é cilíndrico com inserção central, cor castanha. O basidioma medindo $7,0-8,5 \mathrm{~cm}$ de altura (Figura 1).

Agaricus spp. (BRASIL; Bahia: Teixeira de Freitas; Campus X; Silva, L. R.)

Algumas espécies do gênero Agaricus são importantes por serem comestíveis (SINGER, 1986). É um grande gênero com 200 espécies, são saprofíticos, podem ser anuais, geralmente são brancos (WEBSTER; WEBER, 2007). O espécime encontrado possui píleo hemisférico, liso de cor branca com cerca de $5,0 \mathrm{~cm}$ de diametro, o estipe cilindrico central, com anel ambos na cor branca com cerca de 6,0 cm e altura, píleo semiaberto, com lamela na cor salmão. Esporos castanhos com cerca e 8,0-10,0 x 4,0$5,0 \mu \mathrm{m}$ (Figura 1).

\section{Família Sclerodermataceae}

Família composta por 50 espécies e sete gêneros (KIRK et al., 2001), algumas espécies conhecidas como bolas da terra (Scleroderma spp.) e bolas de esporos (Calostoma) (WEBSTER; WEBER, 2007). Por longo período este táxon fez parte de Lycoperdales, ordem dos Gasteromycetes. Os motivos para esta mudança na classificação são as diversas evidências moleculares, com base principalmente no sequenciamento de DNA (BASEIA et al., 2006).

Scleroderma spp. (BRASIL; Bahia: Teixeira de Freitas; Campus X; Silva, L. R.)

Foram coletados seis individuos desse gênero, todos em uma mesma área, por suas caracteristicas macro e miroscopicas, possivelmente são da mesma espécie. Quando maduros medindo cerca de 6,5-10,0 x 4,0-9,0 cm, quando ainda estão fechados $3,5-4,0 \times 3,0-4,0 \mathrm{~cm}$. Os esporos são castanho escuro, circulares com aprencia espinhosa em todo ele, medindo cerca de 12,0-17,0 $\mu \mathrm{m}$ (Figura 1).

Há uma ampla quantidade de caracteres para separar as espécies desse gênero, como se possuem estipe, a espessura. Há também controvérsias quanto às divisões, alguns autores os dividindo em gênero e subgênero (PHOSRI, et al., 2009). Segundo Webster e Weber (2007), essas bolas da terra, aparecem frequentemente no outono, podem ser ectomicorrizas de pinheiros, bétulas, carvalhos e fagus. Possuem aparentemente, uma única camada bastante grossa. Quando o gasteroma está maduro, fazem-se rachaduras na superficie que se abre de forma irregular e os esporos secos, 
ficam com aparencia de uma flor aberta e quando está se abrindo.

\section{Família Polyporaceae}

Dentre as espécies da família Polyporaceae é possível encontrar fungos que apresentam tubos ou poros (VIEIRA et al., 2006). Geralmente crescem em troncos de arvores, podem crescer anualmente (WEBSTER; WEBER, 2007).

Lentinus crinitus (BRASIL; Bahia: Teixeira de Freitas; Campus X; Silva, L. R.)

Foi encontrado apenas um indivíduo dessa espécie, sendo facilmente reconhecido por seu formato umbilicado do píleo, de cor castanho amarelados, com "pelos" eriçados, lamelas castanho alaranjadas, com impressão dos esporos brancas. O píleo medindo cerca de $1,5 \mathrm{~cm}$ de diâmetro, o estipe é central de cor um pouco mais escura, com "pelos", medindo cerca de 2,0 cm de altura (Figura 1).

Pycnoporus sanguineus (BRASIL; Bahia: Teixeira de Freitas; Campus X; Silva, L. R.)

Foi coletado um indivíduo dessa espécie, esse medindo em média $3,0 \times 4,5 \mathrm{~cm}$, de cor vermelho alaranjado, com borda em um tom mais claro, com lamela poroídes, com a mesma cor (Figura 1). Os indivíduos dessa espécie destacam-se pela coloração vermelho alaranjada em seus basidiomas. Nutrem-se saprofiticamente, são decompositores de decomposição branca, pois degradam celulose, a hemicelulose e lignina (MARTINAZZO-PORTZ, 2011). De acordo com Gerrero e Homrich (1999), esta espécie ocorre comumente em locais ensolarados, sobre postes, troncos caídos e madeiras de construção, podendo ser vistos crescendo em plantas vivas ou mortas.

\section{Família Hymenochaetaceae}

Hymenochaetaceae é uma família caracterizada pela presença de estirilpironas que lhes conferem coloração marrom, hifas simples-septadas com septo dolíporo imperfurado, presença ou ausência de setas e por causarem podridão branca na madeira, degradando a lignina, celulose e hemicelulose (LOPES, 2013). Desta família foi identificado apenas um espécime, sendo este identificado em nível de espécie.

Phylloporia chrysita (BRASIL; Bahia: Teixeira de Freitas; Campus X; Silva, L. R.)

Foram coletados cinco indivíduos. Os indivíduos dessa espécie geralmente têm superfície himenial poróide, marrom escura, amplamente aderido em ramos vivos de arbustos e árvores (LOPES, 2013). P. chrysita encontrados estavam em associação com líquens na parte superior do basidioma, medindo de 1,2-1,8 x 3,0-4,0 cm e superfície himenial de cor castanha (Figura 2).

\section{Família Psathyrellaceae}

Pertencente à ordem Agaricales, com 12 gêneros, 746 espécies (KIRK, et al., 2008). A família (anteriormente tratada por nome Coprinaceae) inclui dois grandes gêneros, Coprinus e Psathyrella. Algumas espécies do gênero Psathyrella são comestiveis, também conhecidas por decomposição de lixo e madeira, raramente encontrada parasitando outros fungos ou em esterco. Alguns gêneros podem ser patógenos de humanos e animais (NAGY et al., 2013).

Kirk et al. (2008) explicam que a família Psathyrellaceae, ainda precisa de estudos 
no que se refere a organização de seus gêneros e espécies.

Panaeolus acuminatus (BRASIL; Bahia: Teixeira de Freitas; Campus X; Silva, L. R.)

Os basidiomas pertencentes a essa espécie tem por característica a cor castanha, tanto na estipe como no píleo, este em especial em suas extremidades (na maioria das vezes) é um castanho mais escuro, lamelas escurecidas. Cerca de 3,0-4,0 cm altura, com píleo de 0,7-0,9 mm, os basidiósporos têm cerca de 12,0-15,0 x 5,0-6,0 $\mu \mathrm{m}$ (Figura 2). Kirk et al. (2008) descrevem que o gênero Panaeolus, pode pertencer a família Psathyrellaceae ou a Inocybaceae.

FIGURA 2. (1) (A) Phylloporia chrysita no substrato; (B) Superfície da parte superior do basidioma; (C-D) Superfície himenial. (2) (A) Basidiomas de Panaeolus acuminatus; (B) Basidiósporos. (3) (A) Psathyrella spp. no substrato; (B) Basidioma; (C) Lamelas; (D) Basidiósporos. (4) (A) Basidioma de Inocybe rimosa no substrato; (B) Lamelas; (C) Impressão dos esporos; (D) Basidiósporos. (5) (A-B) Entoloma serrulatum no substrato; (C) Lamelas; (D) Basidiósporos angulares. (6) (A)

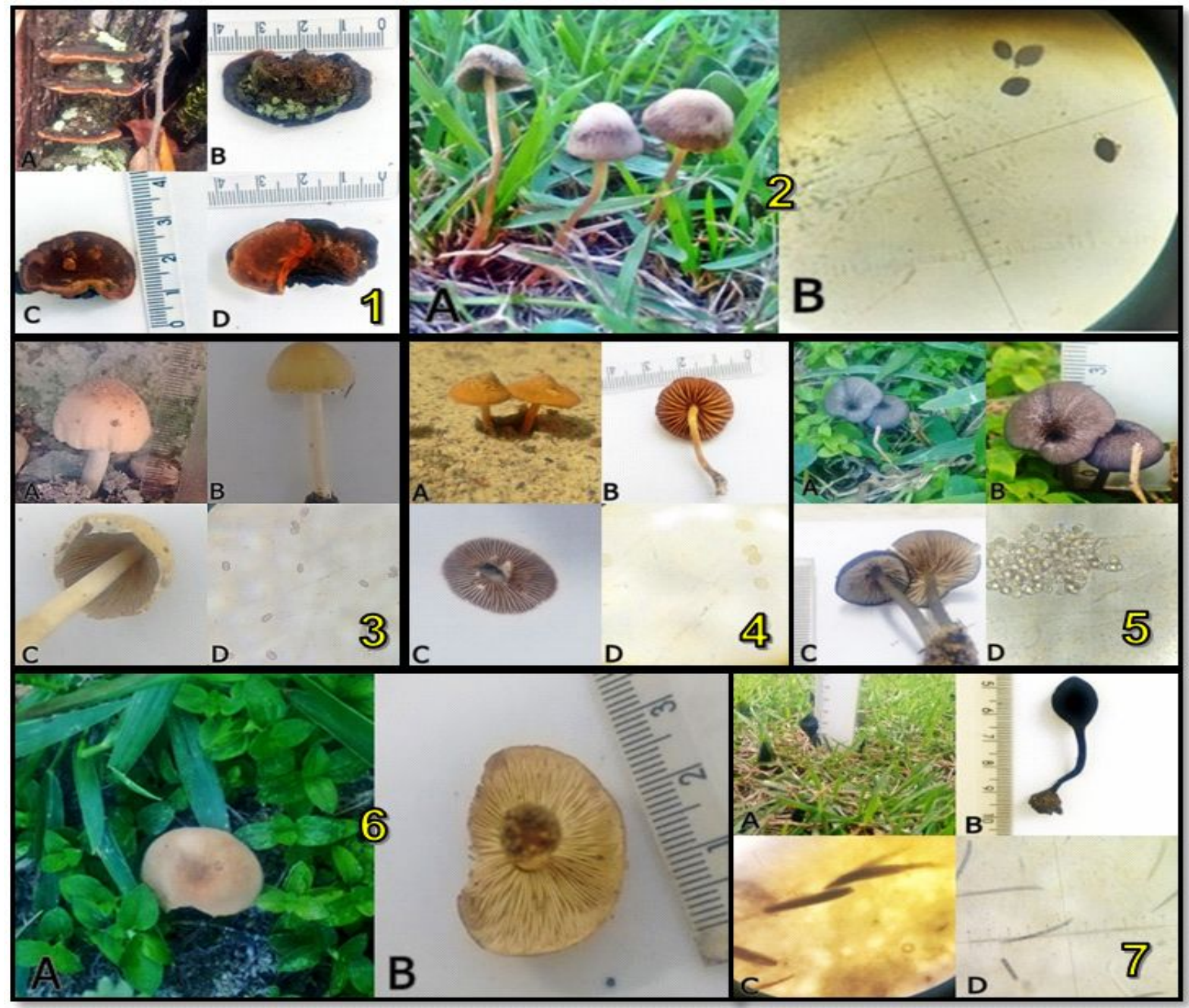

Psathyrella spp. (BRASIL; Bahia: Teixeira de Freitas; Campus X; Silva, L. R.) 
Foram identificados quatro espécimes desse gênero, com píleo de cor marrom claro pálido, medindo cerca de $2,5 \mathrm{~cm}$ de diâmetro, basidioma com altura de $5,5 \mathrm{~cm}$, os basidiósporos são castanhos, medindo 9,0-10,0 x 3,0-5,0 $\mu$ m (Figura 2).

O gênero Psathyrella é representado por 400 espécies de cogumelos em todo o mundo (KIRK et al., 2008). O gênero é caracterizado por píleo hemisférico, marrom claro a marrom escuro. Estipe frágil, branco ou pálido, esporos ovoides a esporos elipsoidais e lisos, de cor castanho escuro (AMANDEEP et al., 2015).

\section{Família Inocybaceae}

Família pertencente a ordem Agarycales, com 13 gêneros e 821 espécies (KIRK et al., 2008). Inocybaceae se faz importante por conta de sua ecologia ectomicorrízica, muitas espécies também são farmacologicamente importantes (MATHENY, 2009).

Inocybe rimosa (BRASIL; Bahia: Teixeira de Freitas; Campus X; Silva, L. R.)

Foram coletados cinco individuos, estes de cor castanha amarelada, são fibrosos, com escâmulas no píleo, que é umbonado no centro (YANGDOL et al., 2016). Estípe com introdução central, possuindo cerca de 3,0-3,5 cm de cumprimento. Píleo com 1,5$2,5 \mathrm{~cm}$ de diâmetro. A impressão dos esporos é de cor marrom, seus esporos são castanhos amarelados medindo 8,0-9,0 x 4,0-5,0 $\mu \mathrm{m}$ (Figura 2).

Foi notado, que esse macrogungo é decorrente, sempre aparecem em grupos, dificilmente apenas um indivíduo no mesmo local. Os membros desse gênero geralmente associam-se a árvores de forma simbionte formando assim relação ectomicorrízica (KROPP; ALBEE-SCOTT, 2010). Essa pode vir a ser confirmada com a espécie encontrada no Campus $\mathrm{X}$. Os espécimes de Inocybe rimosa encontrados estavam em grande número, todos ao redor de uma pequena árvore.

\section{Família Entolomataceae}

Família pertencente a ordem Agaricales, acredita-se que esteja dividido em pelo menos 13 gêneros e cerca de 1.071 espécies (KIRK et al., 2008; TEIXEIRA-SILVA; CORTEZ, 2014). No Brasil, são citadas cerca de 200 espécies de Entolomataceae, distribuídas em dez gêneros, para todas as regiões do país, (TEIXEIRA-SILVA; CORTEZ, 2014).

Microscopicamente, podem ser diferenciados dos demais da ordem pela forma e ornamentação dos basidiósporos, exibem contornos rugoso-angulados, longitudinalmente sulcados a angulados e, macroscopicamente, quando observada a impressão dos esporos, apresentam cor rosa em diferentes tonalidades (SINGER, 1986; TEIXEIRA-SILVA; CORTEZ, 2014).

Entoloma serrulatum (BRASIL; Bahia: Teixeira de Freitas; Campus X; Silva, L. R.)

Pileo convexo ou campanulado, umbilicado, superfície cinza-branca, mais escura e escamosa no ombligo, margem obscura, com lamelas finas. Podem ser encontrados em terra úmida. (NOORDELOOS, 2008). Foram coletados dois espécimes, estes com as características do píleo equivalente ao citado pelo autor. Medindo cerca de a 1,5-3,0 $\mathrm{cm}$ de diâmetro. O estipe cinza azulado cilindrico com inserção central, basiósporo medindo 3,0-4,0 cm e altura. A impressão dos esporos é marrom rosadas, basidiósporos angulares medinho cerca de 9,0-10,0 x 4,0-5,0 $\mu \mathrm{m}$. (Figura 2). 
Entoloma spp. (BRASIL; Bahia: Teixeira de Freitas; Campus X; Silva, L. R.)

O estipe com cor bege pouco mais escura que o píleo, com cerca de $3,0 \mathrm{~cm}$ e altura, píleo campanulado, pouco umbilicado, na cor creme com $2,5 \mathrm{~cm}$ de diâmetro. Impressão de esporos marrom rosado (Figura 2).

\section{Família Geoglossaceae}

Os membros da família Geoglossaceae são geralmente terrícolas, popularmente conhecidos como línguas-da-terra (PEREIRA; BEZERRA 2011), e estão distribuídos em seis gêneros e 48 espécies (KIRK et al. 2008). Apresentam ascomas apoteciais com consistência cartilaginosa a cerosa, medindo até $10 \mathrm{~cm}$ de altura, possuindo ou não um estípite. A coloração dos ascomas pode variar na cor preta, marrom, esverdeada ou amarelada (PEREIRA; BEZERRA 2011).

Geoglossum fallax (BRASIL; Bahia: Teixeira de Freitas; Campus X; Silva, L. R.)

Foram coletados dez indivíduos dessa espécie, todos apresentando ascomas tipicamente pretos, com base fina que se estende até se alargar levemente, esta sendo a parte reprodutiva do ascoma, tendo por diâmetro $0,5-1,0 \mathrm{~cm}$, com 4,5-5,5 cm de altura. Os ascósporos são de cor castanha, medindo 100,0-112,5 x 5,0-7,5 $\mu \mathrm{m}$, os ascósporos possuem cerca de 7-13 septos (Figura 2). Até o ano de 2010 não havia registros de fungos da família Geoglossaceae no Nordeste, apesar dos levantamentos realizados (PEREIRA; BEZERRA 2011), sendo esta espécie (Geoglossum fallax) a primeira registrada no Nordeste do Brasil, segundo Silva e Fortuna (2020).

Com os resultados obtidos pode-se ressaltar a importância de trabalhos de identificação de macrofungos, para que assim os registros de espécies de macrofungos no Extremo Sul aumentem podendo, assim, ter um mapeamento de macrofungos endêmicos, bem como macrofungos anuais da região.

\section{CONCLUSÃO}

A partir das identificações feitas pode-se constatar que no Campus $X$ da Universidade do Estado da Bahia, o filo predominante é o Basidiomycota, com o maior número de espécies identificadas. Constatou-se também que o filo Ascomycota encontra-se representado por uma família de importante registro para o Extremo Sul baiano e para o nordeste.

\section{REFERÊNCIAS}

AMANDEEP, K.; ATRI, N. S.; MUNRUCHI, K. A new species of Psathyrella (Psathyrellaceae, Agaricales) collected on dung from Punjab, India. Environmental \& Applied Mycology. v. 5, n. 2, p. 128-137, 2015. URL: http://www.creamjournal.org/pdf/Cream_5_2_6.pdf

ALMEIDA, T. M.; MOREAU, A. M. S. S.; MOREAU, M. S.; PIRES, M. M.; FONTES, E. O.; GÓES, L. M. Reorganização socioeconômica no Extremo Sul da Bahia decorrente da introdução da cultura do eucalipto. Sociedade \& Natureza. v. 20, n. 2, p. 5-18, 2008. DOI: https://doi.org/10.1590/S1982-45132008000200001

BASEIA, I. G.; SILVA, B. D. B.; LEITE, A. G.; MAIA, L. C. O gênero Calostoma 
(Boletales, Agaricomycetidae) em áreas de cerrado e semi-árido no Brasil. Acta Botânica Brasílica. v. 21, n. 2, 277-280. 2007. DOI: https://doi.org/10.1590/S010233062007000200003

CORTEZ, V. G.; SILVEIRA, R. M. B. New species of Stropharia with hymenial acanthocytes. Mycologia. v. 99, p. 135-138, 2007a. DOI: https://doi.org/10.1080/15572536.2007.11832609

Species of Hypholoma (Fr.) P. Kumm. (Strophariaceae, Agaricales) in Rio Grande do Sul State, Brazil. Acta Botânica Brasilica. v. 21, p. 609-621, 2007b. DOI: https://doi.org/10.1590/S0102-33062007000300008

COSTA, N. L.; J. DIONÍSIO, A.; ANGINONI, I. Influência de fungos micorrízicos vesículo-arbusculares, fontes e doses de fósforo sobre o crescimento de aveia forrageira. Pesquisa Agropecuária Brasileira. v. 24, n. 8, p. 979-986, 1989. URL: https://seer.sct.embrapa.br/index.php/pab/article/view/18386

DENNIS, R. W. G. Lepiota and allied genera in Trinidad, British West Indies. Kew Bulletin. v. 7, p. 459-499, 1952. DOI: https://doi.org/10.2307/4117800

DIDUKH, M. Y. A.; WASSER, S. P.; NEVO, E. Medicinal value of species of the Family Agaricaceae Cohn (Higher Basidiomycetes): Current stage of knowleadge and future perspectives. International Journal of Medicinal Mushrooms. v. 5, p. 133-152, 2003. DOI: https://doi.org/10.1615/InterJMedicMush.v5.i2.30

DOMANN, B, C., POLETO, L., MACCAGNAN, B., CAMASSOLA, M., PAESI, S. Avaliação da atividade antimicrobiana de macrofungo sobre bactérias de importância alimentar. Projeto Pró Amazônia. XXII Encontro de Jovens Pesquisadores. V Mostra Acadêmica de Inovação e Tecnologia. 2015.

ESPOSITO, E.; AZEVEDO, J. L. Fungos: Uma Introdução à Biologia, Bioquímica e Biotecnologia. 2. ed. Caxias do Sul: Educs. 2010. 637 p.

GERRERO, R.; HOMRICH, M. M. Fungos Macroscópicos Comuns no Rio Grande do Sul - Guia para Identificação. 2. ed. Porto Alegre: UFRGS. 1999.

GIMENES, L. J.; MATHEUS, D. R. Fungos Basidiomicetos. Técnicas de Coleta, Isolamento e Subsídios para Processos Biotecnológicos. Instituto de Botânica (IBT). Programa de Pós Graduação em Biodiversidade Vegetal e Meio Ambiente. Curso de Capacitação de Monitores e Educadores. 2010.

GONÇALVES, F. S. Origem dos Antibióticos. Info Escola. 11 Março 2010 [Online]. Disponível em: <http://www.infoescola.com/microbiologia/origem-dos-antibioticos/> Acessado em 10 de outubro de 2016.

GUGLIOTTA, A. M.; CAPELARI, M. Taxonomia de Basidiomicetos. p. 68-102. In: 
BONONI, V. L. R. (Org.). Zigomicetos, Basidiomicetos e Deuteromicetos: Noções Básicas de Taxonomia e Aplicações Biotecnológicas. São Paulo: Instituto de Botânica, SEMA-SP. 1998.

HERNANDEZ, M. C.; GONZÁLES, S. G. M.; HERRERA, G. M. R.; VILARÓ, M. C. V; HERNANDEZ, N. B.; FIGUEROA, S. H.; MEDINA, J. L. O; SUÀREZ, L. C.; GARCIA, A. T. Catálogo e Hongos y Myxomycetes del Jardin Botânico Nacional e Cuba. Editóra Universitária. 2014. 84 p.

KIRK, P. M.; CANNON, P. F.; MINTER, D. W.; STALPERS, J. A. Ainsworth and Bisby's Dictionary of the Fungi. 10. ed. Wallingford: CAB International University Press, Cambridge. 2008.

Dictionary of the Fungi. 9. ed. CABI Publishing, Surrey. 2001.

KROPP, B. R.; ALBEE-SCOTT, S. Inocybe tauensis, a new species from the Samoan Archipelago with biogeographic evidence for a Paleotropical origin. Fungal Biology, v. 114, n. 9, p. 790-796, 2010. DOI: https://doi.org/10.1016/j.funbio.2010.07.005

LOPES, V. F. Revisão de Phylloporia murrill (Hymenochaetaceae) com ênfase em espécies que ocorrem na região Neotropical. 2013. 168 p. Dissertação (Mestrado). Programa de Pós-Graduação em Biologia de Fungos, Algas e Plantas. Universidade Federal de Santa Catarina.

MARQUES, M. B. S. Diversidade e Ecologia dos Macrofungos do Jardim Botânico da Universidade de Coimbra. Porto-Portugal. 2012. 100 p. Dissertação (Mestrado). Faculdade de Ciências da Universidade do Porto.

MARTINAZZO-PORTZ, T. Formulação de substrato para produção de basidiocarpos de Pycnoporus sanguineus. 2011. 80 f. Dissertação (Mestrado em Agronomia) - Universidade Estadual do Oeste do Paraná. Marechal Cândido Rondon.

MATHENY, P. B. A phylogenetic classification of the Inocybaceae. Mcllvainea. v. 18 n. 1, p. 11-22, 2009. URL: https://namyco.org/docs/3_Matheny.pdf

MYCOBANK. The Fungal Website. [online]. Disponível em: <http://www.mycobank.org> Acessado em 04 de julho de 2017.

NAGY, L. G.; WALTHER, G.; HÁZI, J.; VÁGVÖLGYI, C.; PAPP, T. Understanding the evolutionary processes of fungal fruiting bodies: correlated evolution and divergence time in the Psathyrellaceae. Systematic Biology, v. 60, p. 303-317, 2011. DOI: https://doi.org/10.1093/sysbio/syr005

NOORDELOOS, M. E. Entoloma in North America 2: the species described by C. H. PECK. Österreichische Zeitschrift für Pilzkunde. v. 17, 2008. URL: https://www.zobodat.at/pdf/OestZPilz_17_0087-0152.pdf 
PEREIRA, A. B; PUTZKE, J. Famílias e Gêneros de Fungos Agaricales (Cogumelos) no Rio Grande do Sul. Santa Cruz do Sul: EDUNISC. 1990. 188 p.

PEREIRA, L. T.; BEZERRA, J. L. Trichoglossum hirsutum (Geoglossaceae, Ascomycota): primeiro registro para o Nordeste brasileiro. Sitientibus Série Ciências Biológicas, v. 11, n. 1, p. 99-101, 2011. DOI: https://doi.org/10.13102/scb145

PETERSEN, J. H.; GABA, A.; LÆSSSØE, T. MycoKey 4.0. The Mycological Identification Site. MycoKeys Online Morphing Mushroom Identifier (MMIB) Quickstart. [online]. Disponível em <http://www.mycokey.com/newMycoKeySite/MycoKeyldentQuick.html> Acessado em 18 de julho de 2017.

PHOSRI, C.; MARTÍN, M. P.; WATLING, R.; JEPPSON, M.; SIHANONTH, P. Molecular phylogeny and re-assessment of some Scleroderma spp. (Gasteromycetes). Anales del Jardín Botánico del Madrid, v. 66, p. 83-91, 2009. DOI: https://doi.org/10.3989/ajbm.2199

PIRES, E. Z.; DALBOSCO, E. Z.; GONÇALVES, M. J.; TONINI, R. C. G. Biodiversidade de basidiomicetos encontrados em um fragmento de Floresta Ombrófila Mista. Ambiência Guarapuava. v. 10, n. 2, p. 489-496, 2014. URL: https://revistas.unicentro.br/index.php/ambiencia/article/viewFile/1459/2279

PROINFRA (Pró-Reitoria de Infraestrutura). Universidade do Estado da Bahia (UNEB). Setor de Arquitetura e Urbanismo. [online]. Disponível: <https://portal.uneb.br/proinfra/> Acessado em 13 de julho de 2017.

PUCCINELLI, C. Marasmius (Basidiomycota - Marasmiaceae) do Parque Estadual das Fontes do Ipiranga (PEFI), São Paulo, SP, Brasil. São Paulo. 2007. 109 p. Dissertação (Mestrado). Biodiversidade Vegetal e Meio Ambiente. Instituto de Botânica da Secretaria do Meio Ambiente.

ROTHER, M. S.; SILVEIRA, R. M. B. Família Agaricaceae (Agaricales, Basidiomycota) no Parque estadual de Itapuã, Viamão, Rio Grande do Sul, Brasil. Revista Brasileira de Biociências. v. 6, n. 3, p. 259-268, 2008. URL: http://hdl.handle.net/10183/23213

SILVA, L. R.; FORTUNA, J. L. First record of Geoglossum fallax (Geoglossaceae, Ascomycota) in the Southernmost region of the state of Bahia, Northeastern Brazil. Bioscience Journal, v. 36, n. 2, p. 602-605, 2020. DOI: https://doi.org/10.14393/BJv36n2a2020-42178

SILVA, M. A. B. Aspectos sobre a ecologia e taxonomia de fungos poliporoides (Basidiomycota) da Mata Atlântica de Santa Catarina, Brasil. Florianópolis. 2013. 66 p. Dissertação (Mestrado). Centro de Ciências Biológicas. Universidade Federal de Santa Catarina.

SILVA, P. S; CORTEZ, V. G; SILVEIRA, R. M. B. Strophariaceae (Agaricales, 
Basidiomycota) no Parque Estadual do Itapuã, Viamão, Rio Grande do Sul: chave para identificação das espécies. Revista Brasileira de Biociências. v. 6, n. 3, p. 253-258, 2008. URL: http://www.ufrgs.br/seerbio/ojs/index.php/rbb/article/view/923/824

SILVA, R. R.; COELHO, G. D. Fungos: Principais Grupos e Aplicações Biotecnológicas. São Paulo: Instituto de Botânica. Programa de Pós Graduação em Biodiversidade Vegetal e Meio Ambiente. Curso de Capacitação de Monitores e Educadores. 2006. 20 p.

SINGER, R. The Agaricales in Modern Taxonomy. 4. ed. Koenigstein: Koeltz Scientific Books. 1986. 981 p.

SPECIES 2000. Integrated Taxonomic Information System (ITIS). Catalogue of Life. [online]. Disponível em: <http://www.catalogueoflife.org/col> Acessado em 11 de julho de 2017.

TEIXEIRA-SILVA, M. A.; CORTEZ, V. G. Entolomataceae (Agaricales, Basidiomycota) no parque Estadual de São Camilo, Palotina-PR. Curitiba. 2014. 148 p. Departamento de Botânica. Universidade Federal do Paraná.

TERÇARIOLI, G. R.; PALEARI, L. M.; BAGAGLI, E. O Incrível Mundo dos Fungos. São Paulo: UNESP. 2010. 128 p.

VELLINGA, E. C. Chlorophyllum and Macrolepiota (Agaricaceae) in Australia. Australian Systematic Botany, v. 16, p. 361-370, 2003. DOI: https://doi.org/10.1071/SB02013

VIEIRA, I. M.; ROCHA, M. H.; CUNHA, E. B.; KADOWAKI, M. K.; OSAKU, C. A. Basidiomicetos da cidade de Cascavel - Oeste do Paraná - e suas aplicações em Biotecnologia. Estudos Biologia, v. 28, n. 65, p. 21-31, 2006. DOI: http://dx.doi.org/10.7213/reb.v28i65.22164

WEBSTER, J.; WEBER, R. Introduction to Fungi. Cambridge: University Press. 2007. $841 \mathrm{p}$.

YANGDOL, R.; KUMAR, S.; LALOTRA, P.; SHARMA, Y. P. Two species of Inocybe from Trans-Himalayan Ladakh (J\&K), India. Current Research in Environmental \& Applied Mycology, v. 6, n. 4, p. 305-311, 2016. DOI: https://doi.org/10.5943/cream/6/4/9 